\section{EFFECTS OF PEMBROLIZUMAB ON THE TUMOR MICROENVIRONMENT (TME) AFTER ONE PRESURGERY TREATMENT CYCLE IN PATIENTS WITH TRIPLE- NEGATIVE BREAST CANCER (TNBC): PHASE 1B KEYNOTE-173 STUDY}

\begin{abstract}
${ }^{1}$ Peter Schmid*, ${ }^{2}$ Yeon Hee Park, ${ }^{3}$ Eva Muñoz-Couselo, ${ }^{4}$ Sung-Bae Kim, ${ }^{5}$ Joohyuk Sohn, ${ }^{6}$ Seock-Ah Im, ${ }^{7}$ Esther Holgado, ${ }^{8}$ Theodoros Foukakis, ${ }^{9}$ Sherko Kümmel, ${ }^{10}$ Rebecca Dent, ${ }^{11}$ Yuan Sun, ${ }^{11}$ Lingkang Huang, ${ }^{11}$ Jennifer Yearley, ${ }^{11}$ Petar Jelinic, ${ }^{11}$ Vassiliki Karantza, ${ }^{3}$ Javier Cortés. ${ }^{1}$ Centre for Experimental Cancer Medicine, London, UK; ${ }^{2}$ Samsung Medical Center, Seoul, Korea, Republic of; ${ }^{3}$ Vall d'Hebron Institute of Oncology (VHIO), Barcelona, Spain; ${ }^{4}$ Asan Medical Center, University of Ulsan College of Medicine, Seoul, Korea, Republic of; ${ }^{5}$ Yonsei Cancer Center, Yonsei University College of Medicine, Seoul, Korea, Republic of; ${ }^{6}$ Seoul National University Hospital, Cancer Research Institute, Seoul National University College of Medicine, Seoul, Korea, Republic of; 'Ramón y Cajal University, Madrid, Spain; ${ }^{8}$ Karolinska Institutet and Breast Cancer Center, Theme Cancer, Karolinska University Hospital, Solna, Sweden; ${ }^{9}$ Kliniken Essen-Mitte, Essen, Germany; ${ }^{1}$ International Breast Cancer Center Quironsalud Group, Singapore, Singapore; ${ }^{11}$ Merck and Co., Inc., Kenilworth, NJ, USA
\end{abstract}

Background In the phase 3 KEYNOTE-522 trial, neoadjuvant pembrolizumab + chemotherapy followed by adjuvant pembrolizumab monotherapy resulted in a statistically significant improvement in pathologic complete response (pCR) and event-free survival, compared with neoadjuvant chemotherapy alone, in patients with early-stage TNBC. In the phase $1 \mathrm{~b}$ KEYNOTE-173 (NCT02622074) trial-another neoadjuvant pembrolizumab+chemotherapy trial-we evaluated TNBC biopsy samples at baseline and collected after one cycle of neoadjuvant pembrolizumab, before the initiation of chemotherapy, to explore the features within the TME at both timepoints that might be potentially predictive of clinical response and the effects of a single cycle of pembrolizumab on cell populations within the TME.

Methods Twenty paired samples (baseline and obtained following one cycle of pembrolizumab before the initiation of chemotherapy) were included. Multiplex immunohistochemistry analyzed deconvoluted cell fractions by spatial localization (tumor compartment, stromal compartment, or total tumor) using three 6-plex panels: T cell (CD3/CD8/FoxP3/Ki67/granzyme B/PD-1), myeloid cell (CD68/CD163/MHCII/arginase/ CD33/CD11c), and natural killer cell (CD16/CD56/CD11b/ CD20/CD3/CD45). Area under the receiver operating characteristic (AUROC) was used to assess associations between immune subsets and pCR. Analyses were descriptive, with topranked findings reported, and were deemed hypothesis generating; no claims of statistical significance are made.

Results At baseline, 6 of 75 evaluated immune subsets (counting different compartments) showed 95\% CIs of AUROC not crossing 0.5 with pCR. These include some myeloid cell populations within the tumor compartment (AUROC, 95\% CI), specifically $\mathrm{CD}_{11 \mathrm{c}^{+}}$(macrophage and dendritic cell [DC]:

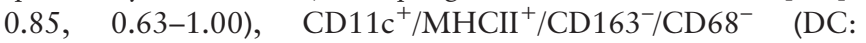
$0.76,0.53-0.99), \quad \mathrm{CD} 11 \mathrm{c}^{+} / \mathrm{MHCII}^{-} / \mathrm{CD}^{-} 63^{-} / \mathrm{CD}^{-} 8^{-}$(nonactivated/immature DC: $0.8,0.54-1.00)$, and $\mathrm{CD} 11 \mathrm{c}^{+} / \mathrm{CD} 163^{+}$ (M2 macrophage: $0.77,0.55-0.99)$. Other associations with pCR included baseline $\mathrm{CD} 11 \mathrm{c}^{+} / \mathrm{MHCII}^{-} / \mathrm{CD} 163^{-} / \mathrm{CD}^{-} 8^{-}$(nonactivated/immature DC) within the total tumor (AUROC, 0.76 ; 95\% CI, 0.51-1.00) and the baseline ratio of CD11c/ CD3 within the tumor compartment (AUROC, 0.75; 95\% CI, 0.52-0.98). Although T-cell associations were relatively weak, specific CD8 subsets, especially $\mathrm{CD}^{+} /$granzyme $\mathrm{B}^{+} / \mathrm{Ki} 67^{+}$, showed a trend toward association. Negative correlations between change from baseline and baseline values were observed; therefore, baseline detrending was applied to change from baseline values. One immune subset showed a negative association trend between change from baseline and pCR after baseline detrending: $\mathrm{CD}_{163}{ }^{+} / \mathrm{MHCII}^{+}$(DC3) within the stroma (AUROC, 0.2; 95\% CI, 0.0-0.42).

Conclusions Although the sample size in this exploratory analysis was small $(n=20)$, myeloid cell populations within the tumor compartment at baseline show a promising association trend, as evaluated by AUROC, with pCR after neoadjuvant pembrolizumab + chemotherapy in early-stage TNBC. Changes in immune subsets following one cycle of pembrolizumab were not strongly associated with pCR.

Trial Registration ClinicalTrials.gov, NCT02622074

Ethics Approval The study protocol and all amendments were approved by the relevant institutional review board or ethics committee at each study site.

Consent All patients provided written informed consent to participate in the clinical trial.

http://dx.doi.org/10.1136/jitc-2021-SITC2021.338 